\title{
QUEEN'S
UNIVERSITY
BELFAST
}

\section{Millimetre wave dielectric characterisation of multilayer LTCC substrate}

Zelenchuk, D., Fusco, V., Breslin, J., \& Keaveney, M. (2015). Millimetre wave dielectric characterisation of multilayer LTCC substrate. In Proceedings of the 45th European Microwave Conference (pp. 1033-1036). Institute of Electrical and Electronics Engineers Inc.. https://doi.org/10.1109/EuMC.2015.7345943

Published in:

Proceedings of the 45th European Microwave Conference

Document Version:

Peer reviewed version

Queen's University Belfast - Research Portal:

Link to publication record in Queen's University Belfast Research Portal

\section{Publisher rights}

( 2016 IEEE. Personal use of this material is permitted. Permission from IEEE must be obtained for all other uses, in any current or future media, including reprinting/republishing this material for advertising or promotional purposes, creating new collective works, for resale or redistribution to servers or lists, or reuse of any copyrighted component of this work in other works.

\section{General rights}

Copyright for the publications made accessible via the Queen's University Belfast Research Portal is retained by the author(s) and / or other copyright owners and it is a condition of accessing these publications that users recognise and abide by the legal requirements associated with these rights.

Take down policy

The Research Portal is Queen's institutional repository that provides access to Queen's research output. Every effort has been made to ensure that content in the Research Portal does not infringe any person's rights, or applicable UK laws. If you discover content in the Research Portal that you believe breaches copyright or violates any law, please contact openaccess@qub.ac.uk. 


\section{Millimetre wave dielectric chartacterisation of multilayer LTCC substrate}

\author{
Dmitry Zelenchuk, Vincent Fusco \\ ECIT, Queen's University Belfast, \\ Belfast, United Kingdom \\ d.zelenchuk@qub.ac.uk,v.fusco@qub.ac.uk
}

\author{
James Breslin, Mike Keaveney \\ Analog Devices Inc., \\ Limerick, Ireland
}

\begin{abstract}
The paper reports on the mm-wave characterization of a low temperature co-fired ceramic (LTCC) substrate. A substrate integrated resonator (SIW) method is presented for robust extraction of both permittivity and loss tangent of the substrate. The data obtained allow full characterization of the substrate in the $71 \mathrm{GHz}-95 \mathrm{GHz}$ frequency range suitable for accurate modelling of $\mathbf{E}$-and $\mathrm{W}$-band printed circuits.
\end{abstract}

Keywords-dielectric characterisation, LTCC; mm-wave communications

\section{INTRODUCTION}

Millimeter-wave communications have found many applications in recent years with on-chip V-band systems [1], Eband backhaul systems [2] and W-band automotive sensors [3] being most prominent examples. The onset of $5 \mathrm{G}$ communications poses even greater emphasis on development of compact and efficient integrated mm-wave devices [4].

For accurate modelling and design of the devices, it is very important to know the parameters of the materials and have an ability to conduct non-destructive tests on the same substrate.

Recently substrate integrated waveguides have been proposed as means of testing the dielectric properties of PCB substrates [5]-[7]. The method has been shown to be accurate up to mm-wave for low dielectric constant substrate.

In recent years LTCC process has become widely used for mm-wave applications due to stable permittivity, good tolerance of the process and easy integration with active components [8][10]. For LTCC substrates with high permittivity direct translation of the previous SIW cavities designs, e.g. from [5], leads to prohibitively small dimensions. In this paper SIW resonator method has been extended for characterization of a multilayer LTCC substrate with high permittivity.

\section{DESIGN}

\section{A. Theory}

A substrate integrated waveguide rectangular cavity is shown in Fig. 1. It has been shown in [11], [12] that both propagation and attenuation constants of a SIW waveguide are equivalent to the quantities of a rectangular waveguide with effective width $a_{e f f}$,

$$
a_{e f f}=a-1.08 \frac{d^{2}}{s}+0.1 \frac{d^{2}}{a}
$$

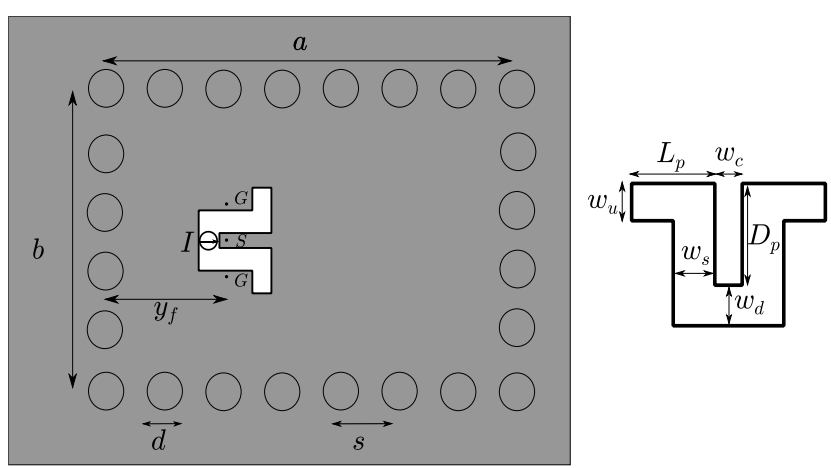

Fig. 1. Layout of a rectangular substrate integrated waveguide resonator fed with GSG-probes through u-shaped aperture.

Additionally the resonant frequency of the $\mathrm{TE}_{\mathrm{mn} 0}$ mode of a rectangular SIW resonator is obtained from:

$$
f_{m n 0}=\frac{c}{2 \pi \sqrt{\varepsilon_{r}}} \sqrt{\left(\frac{m \pi}{a_{e f f}}\right)^{2}+\left(\frac{n \pi}{b_{e f f}}\right)^{2}}
$$

where $c$ is the velocity of light in vacuum and $\varepsilon_{r}$ is the relative permittivity of the substrate.

As described in [5], [13] there are three major loss mechanisms associated with SIW: dielectric loss, conductor loss and radiation loss. Therefore, the unloaded quality factor $Q_{U}$ of the cavity is:

$$
\frac{1}{Q_{U}}=\frac{1}{Q_{C}}+\frac{1}{Q_{D}}+\frac{1}{Q_{\text {rad }}}
$$

Here $Q_{C}$ is the quality factor associated with the conductor loss, whereas $Q_{D}$ is the quality factor associated with the dielectric loss, whereas $Q_{\text {rad }}$ is the quality factor due to radiation loss, which is in case of SIW cavity is manifested by leakage through the via walls. The latter can be designed as a negligible contributor subject to the sufficient via density [5], [13], [14]. $Q_{C}$ can be determined by the cavity geometry, permittivity of the dielectric and the surface resistance of the metal $R_{S}$, whereas $Q_{D}$ is reciprocal to the dielectric loss tangent $\tan \delta[15]$.

LTCC process metallization consists of copper on the outer layers and metal with conductivity twice as low as copper for 
inner layers and vias, the electromagnetic properties of the metals are well known.

The quality factor $Q_{C}$ for the $\mathrm{TE}_{1 \mathrm{n} 0}$ mode of a rectangular cavity with different wall, top and bottom metallization with surface resistance $\mathrm{R}_{\mathrm{s}_{\mathrm{w}}}, \mathrm{R}_{\mathrm{s}_{\mathrm{t}}}$ and $\mathrm{R}_{\mathrm{s}_{\mathrm{b}}}$ respectively reads as [5]:

$$
\begin{aligned}
Q_{C_{d}}=\frac{k_{c}^{3} h \eta \pi^{-2}}{2 R_{s_{w}}\left(2 n^{2} b_{e f f}^{-3} h+2 h a_{e f f}^{-3}\right)} & \\
& +\frac{k_{c}^{3} h \eta \pi^{-2}}{\left(R_{s_{t}}+R_{s_{b}}\right)\left(n^{2} b_{e f f}^{-2}+a_{e f f}^{-2}\right)}
\end{aligned}
$$

where

$$
k_{c}=\sqrt{\left(\frac{\pi}{a_{e f f}}\right)^{2}+\left(\frac{n \pi}{b_{e f f}}\right)^{2}}
$$

$h$ is the substrate thickness, and $\eta=120 \pi / \sqrt{\varepsilon_{r}}$ Ohm

We note that for a rectangular cavity with reduced height, conductor losses in the sidewalls (reciprocal of the first term of (4)) are smaller than those in the top and bottom walls (reciprocal of the second term of (4)).

\section{B. Extraction of Dielectric Properties}

Once the resonant frequency and the quality factor of the resonator have been measured, the substrate permittivity $\varepsilon_{\mathrm{r}}$ and the loss tangent $\tan \delta$ can be obtained

$$
\begin{gathered}
\varepsilon_{r, \text { rect }}=\left(\frac{c}{2 \pi f_{m n 0}}\right)^{2}\left(\left(\frac{m \pi}{a_{\text {eff }}}\right)^{2}+\left(\frac{n \pi}{b_{\text {eff }}}\right)^{2}\right)^{2} \\
\tan \delta=\frac{1}{Q_{U}}-\frac{1}{Q_{C_{d}}}
\end{gathered}
$$

Importantly, it follows from (5) that the extracted permittivity does not depend on the thickness of the substrate and the metal loss is defined by closed-form accurate expression (4). This makes the presented approach preferable to other planar techniques like microstrip or stripline resonator measurement, where individual designs and subsequent extractions are necessary for each substrate thickness. The radiation loss is discarded in (5) as the proposed method assumes design with negligible leakage.

\section{SIW cavity design on LTCC substrate}

At mm-wave frequencies there are technological limitations posed on the design of the SIW cavities. These are particularly prominent for higher permittivities of LTCC substrates. Namely the size of a cavity at fundamental mode becomes close to the via period, which can limit applicability of the effective width formula (1) and hence the method itself. It is also important to incorporate a launching mechanism that does not substantially alter the fields distribution in the cavity.

For the particular substrate under test the manufacturer specified nominal values of the permittivity as 7.8 and loss tangent as 0.0014 . The via diameter was set to 100 microns with period of 250 microns. As the substrate was subsequently planned for other multilayer applications the two-layer dielectric with thicknesses of $0.22 \mathrm{~mm}$ and $0.33 \mathrm{~mm}$ was used, see Fig. 2 .

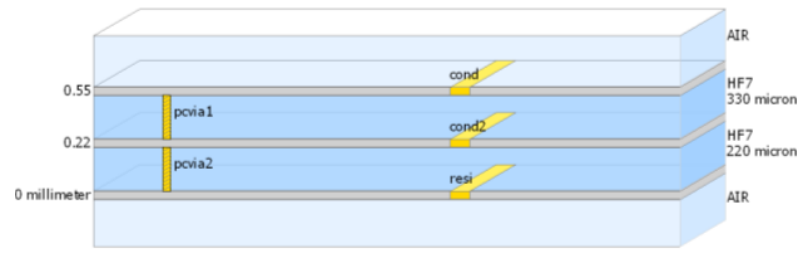

Fig. 2. Layer mapping ("cond" - top metal M1, "cond2"- middle metal "M2", "resi" - bottom metal M3).

First, it is important to choose correct dimensions of the launching structure. Here we use the u-shaped slot, see Fig. 1, as it does allow direct measurement with 50-Ohm GSG probe and eliminates the need for additional calibration of a cable or a transition extensions. In order to accommodate 150 micron GSG probe the dimensions of the $\mathrm{u}$-shaped slot were chosen as $w_{s}=$ $w_{d}=w_{u}=50 \mu \mathrm{m}, w_{c}=L_{p}=100 \mu \mathrm{m}, D_{p}=250 \mu \mathrm{m}$. It is difficult to make the shape smaller due to dimensions of the probe as well as due to etching tolerances of the process.

The structure is not electrically small in comparison to the wavelength in the dielectric $\lambda_{0}=c / f \sqrt{\varepsilon_{r}}$, which is at $90 \mathrm{GHz}$ equals to $1.19 \mathrm{~mm}$. Obviously, at fundamental $\mathrm{TE}_{110}$ mode the launcher will introduce significant distortion to the field distribution, that would be difficult to define as a lumped reactance, see [5]. In order to reduce the effect and a higher order resonance should be chosen.

The resonators were designed to operate at $\mathrm{TE}_{130}$ mode with estimated value of permittivity of 7.8 , see Fig. 3 . The position of the launcher was chosen as $y_{f}=7 / 16 a_{e f f}$, which results in the coupling coefficient between 0.1 and 1 , recommended in [16] for reduction of measurement errors.

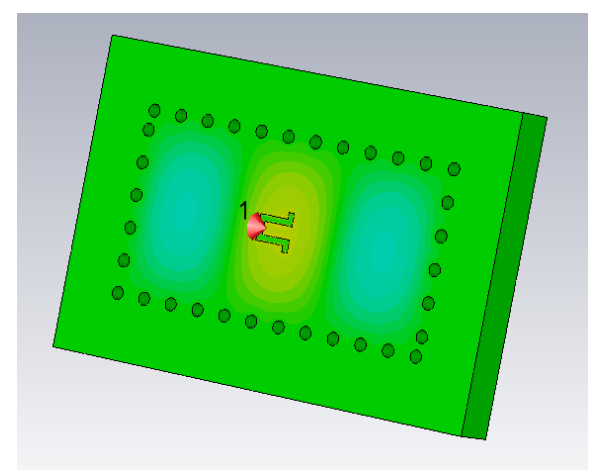

Fig. 3. The field distribution of the $\mathrm{TE}_{13}$ resonance mode in an SIW resonator.

There were 8 SIW resonators designed to characterize the substrate dielectric at frequencies from $71 \mathrm{GHz}$ to $95 \mathrm{GHz}$. The width of all the resonators $b$ is kept as $1.4 \mathrm{~mm}$, and lengths $a$ of the resonators are $2.77 \mathrm{~mm}, 2.64 \mathrm{~mm}, 2.52 \mathrm{~mm}, 2.32 \mathrm{~mm}$, $2.15 \mathrm{~mm}, 1.98 \mathrm{~mm}, 1.94 \mathrm{~mm}$, and $1.91 \mathrm{~mm}$. Knowing the length and width of the resonators as well as the via parameters and metal losses one can establish the permittivity of the substrate from measured $\mathrm{S}_{11}$ as discussed above. 


\section{EXPERIMENTAL RESULTS}

The groups of the 8 resonators have been placed in 4 rows: two from each side of the sample. As the board has two dielectric layers with thicknesses of $0.22 \mathrm{~mm}$ and $0.33 \mathrm{~mm}$, the arrangement is to test each variation of the material from layer to layer as well as across the layers. The metallization of the outer metal layers is copper $\sigma_{\text {outer }}=5.8 \times 10^{7} \mathrm{~S} / \mathrm{m}$ and of the inner metal layer (bottom of the resonators) and of the vias is $\sigma_{\text {inner }}=2.98 \times 10^{7} \mathrm{~S} / \mathrm{m}$.

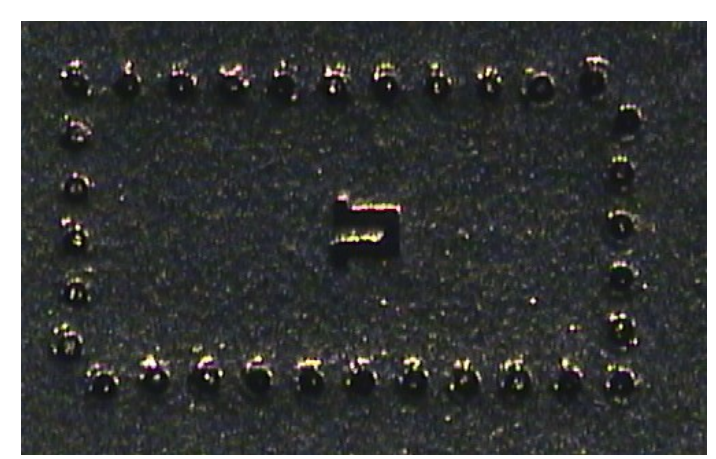

Fig. 4. Photo of an SIW resonator under test.

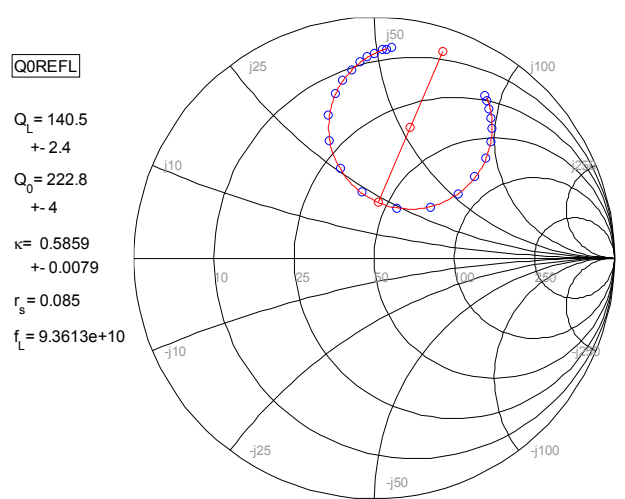

Fig. 5. Smith chart of measured resonator with length of $1.91 \mathrm{~mm}$.

The measurements were carried out on a Cascade Microtech probe station connected to Agilent PNA E8631C. Each resonator was measured with Cascade Infinity GSG 150 micron pitch probe. An SIW resonator under test is shown in Fig. 4. The measured $\mathrm{S}_{11}$ for a resonator with length of $1.91 \mathrm{~mm}$ is demonstrated in Fig. 5. One can observe no significant measurement uncertainties as all the measured points lie on the fitted circle [16].

The extraction of the quality factor is done following procedures given in [5], [17]. As shown in in Fig. 6, the unloaded quality factor $Q_{U}$ extracted from the measurements varies across the samples and close to 200 , whereas calculated quality factor due to conductor losses $Q_{C}$ steadily increase with frequency from 500 to 600 .

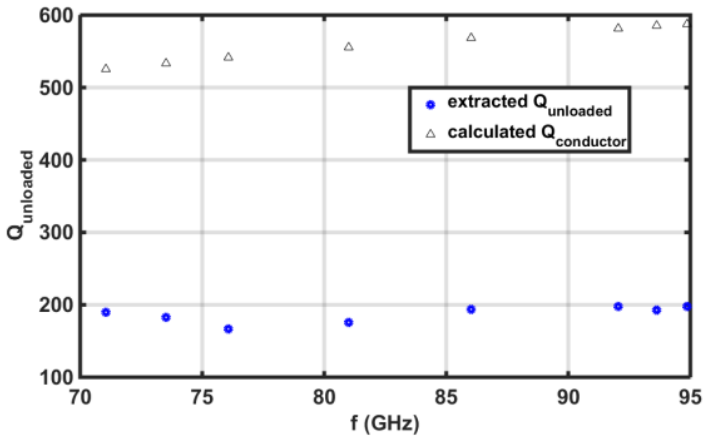

Fig. 6. Quality factor of the SIW resonators placed on $0.22 \mathrm{~mm}$ thick substrate.

The extracted permittivity and loss tangent are plotted in Fig. 7 and Fig. 8. The four batches of the resonators can be separated into two groups as 'siw' and 'asiw' batches are on the bottom 0.22 mm thick layer and the batches 'bsiw' and 'csiw' are on the top $0.33 \mathrm{~mm}$ thick layer. The batches on the same side of the board are shifted $5 \mathrm{~cm}$ from each other in order to test consistency of the parameters across the board.

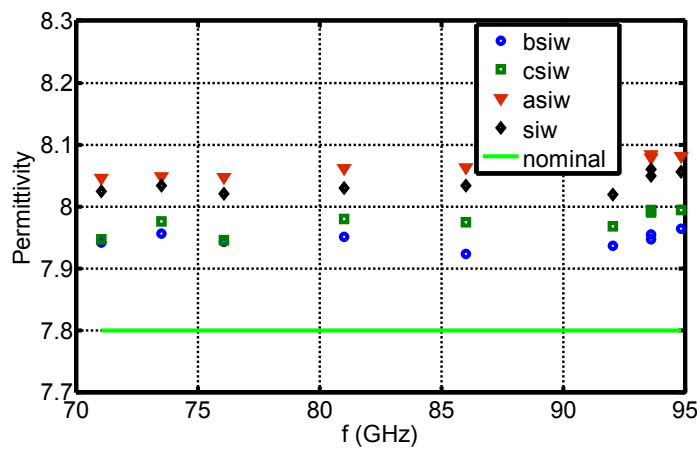

Fig. 7. Extracted permittivity.

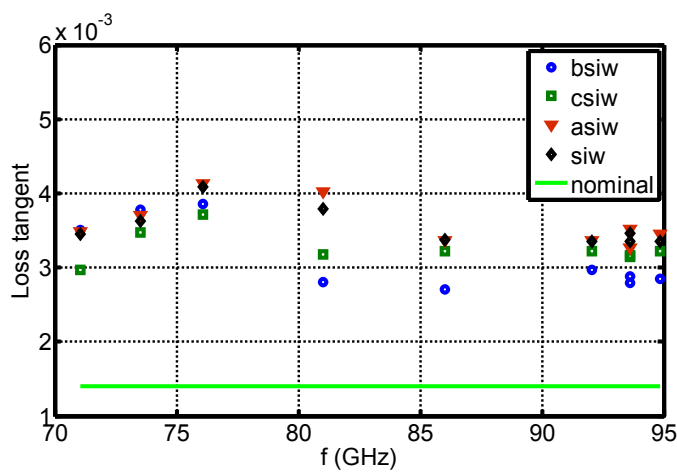

Fig. 8. Extracted loss tangent.

It follows from the measured permittivity that resonators that placed on the $0.33 \mathrm{~mm}$ thick layer have average value of 8.03 whereas at $0.22 \mathrm{~mm}$ thick layer the value is 7.95 . It has to be noted that the permittivity is stable with frequency across the whole 71-95 GHz frequency range. 
The loss tangent varies between 0.003 and 0.004 , which is significantly higher than nominal value of 0.0014 . The discrepancy requires further investigation as neither radiation nor metal losses were found to accommodate the difference.

Comparison of the results from the batches from the same side of the board one may also conclude that there is very little variation of the permittivity and loss tangent across the board. The latter finding supports the choice of the material as suitable candidate for mm-wave applications.

\section{CONCLUSION}

A novel SIW resonator method has been proposed for characterization of multilayer LTCC substrate at mm-waves. There were four batches of the resonators designed and measured. The extracted permittivity and loss tangent are consistent across the batches in the frequency range from 71 $\mathrm{GHz}$ to $95 \mathrm{GHz}$. The permittivity is found to be stable across the frequency range with average value of 7.95 at $0.22 \mathrm{~mm}$ thick substrate and 8.03 at $0.33 \mathrm{~mm}$ thick substrate.

\section{ACKNOWLEDGMENT}

The authors are grateful to Analog Devices Inc for facilitating the manufacturing process and TDK for manufacturing of the samples and providing the data on the manufacturing process.

\section{REFERENCES}

[1] Y. P. Zhang and D. Liu, "Antenna-on-Chip and Antenna-in-Package Solutions to Highly Integrated Millimeter-Wave Devices for Wireless Communications," IEEE Trans. Antennas Propag., vol. 57, no. 10, pp. 2830 2841, Oct. 2009.

[2] J. A. Zhang, W. Ni, J. Matthews, C.-K. Sung, X. Huang, H. Suzuki, and I. Collings, "Low latency integrated point-to-multipoint and e-band point-topoint backhaul for mobile small cells," in 2014 IEEE International Conference on Communications Workshops (ICC), 2014, pp. 592-597.

[3] B.-H. Ku, O. Inac, M. Chang, and G. M. Rebeiz, "75-85 GHz flip-chip phased array RFIC with simultaneous 8-transmit and 8-receive paths for automotive radar applications," 2013 IEEE Radio Freq. Integr. Circuits Symp. pp. 371-374, Jun. 2013.

[4] J. G. Andrews, S. Buzzi, W. Choi, S. V. Hanly, A. Lozano, A. C. K. Soong, and J. C. Zhang, "What will 5G be?," IEEE J. Sel. Areas Commun., vol. 32, no. 6, pp. 1065-1082, Jun. 2014.
[5] D. E. Zelenchuk, V. Fusco, G. Goussetis, A. Mendez, and D. Linton, "Millimeter-Wave Printed Circuit Board Characterization Using Substrate Integrated Waveguide Resonators," IEEE Trans. Microw. Theory Tech., vol. 60 , no. 10 , pp. $3300-3308$, Oct. 2012

[6] X. C. Zhu, W. Hong, K. Wu, K. Da Wang, L. S. Li, Z. C. Hao, H. J. Tang, and J. X. Chen, "Accurate characterization of attenuation constants of substrate integrated waveguide using resonator method," IEEE Microw. Wirel. Components Lett., vol. 23, no. 12, pp. 677-679, 2013.

[7] A. E. Engin, "Extraction of Dielectric Constant and Loss Tangent Using New Rapid Plane Solver and Analytical Debye Modeling for Printed Circuit Boards," IEEE Trans. Microw. Theory Tech., vol. 58, no. 1, pp. 211-219, Jan. 2010

[8] W. Byun, B. S. Kim, K. S. Kim, K. C. Eun, M. S. Song, R. Kulke, O. Kersten, G. Möllenbeck, and M. Rittweger, "Design of vertical transition for $40 \mathrm{GHz}$ transceiver module using LTCC technology," in Proceedings of the 37th European Microwave Conference, EUMC, 2007, no. October, pp. 1353 1356

[9] D. G. Kam, S. Member, D. Liu, A. Natarajan, S. Reynolds, H. Chen, B. A. Floyd, and A. Abstract, "LTCC Packages With Embedded Phased-Array Antennas for $60 \mathrm{GHz}$ Communications," vol. 21, no. 3, pp. 142-144, 2011.

[10] J. Xu, Z. N. Chen, X. Qing, and W. Hong, "Bandwidth Enhancement for a $60 \mathrm{GHz}$ Substrate Integrated Waveguide Fed Cavity Array Antenna on LTCC,' IEEE Trans. Antennas Propag., vol. 59, no. 3, pp. 826-832, Mar. 2011.

[11]M. Bozzi and L. Perregrini, "Modeling of Conductor, Dielectric, and Radiation Losses in Substrate Integrated Waveguide by the Boundary IntegralResonant Mode Expansion Method," IEEE Trans. Microw. Theory Tech., vol. 56, no. 12, pp. 3153-3161, Dec. 2008

[12]F. $\mathrm{Xu}$ and $\mathrm{K} . \mathrm{Wu}$, "Guided-wave and leakage characteristics of substrate integrated waveguide," IEEE Trans. Microw. Theory Tech., vol. 53, no. 1, pp. 66-73, Jan. 2005

[13] M. Bozzi, M. Pasian, L. Perregrini, and K. Wu, "On the losses in substrateintegrated waveguides and cavities," International Journal of Microwave and Wireless Technologies, vol. 1, no. 05. p. 395, 02-Sep-2009.

[14] W. Che, D. Wang, K. Deng, and Y. L. Chow, "Leakage and ohmic losses investigation in substrate-integrated waveguide," Radio Sci., vol. 42, no. 5, Oct. 2007.

[15]D. M. Pozar, Microwave Engineering, 2nd ed. John Wiley \& Sons, 1997

[16]D. Kajfez, "Random and systematic uncertainties of reflection-type Qfactor measurement with network analyzer," IEEE Trans. Microw. Theory Tech., vol. 51, no. 2, pp. 512-519, 2003.

[17]D. Kajfez, Q Factor Measurements Using MATLAB. Norwood, MA 02062 Artech House, 2011, p. 189. 